\title{
STRONG INTERACTION EFFECTS IN SCALAR TOP PRODUCTION
}

\author{
M. DREES \\ Lab de Physique Mathematique, Univ. de Montpellier II, France \\ O.J.P. ÉBOLI \\ Inst. de Física Téorica, Univ. Estadual Paulista, São Paulo, Brazil
}

\begin{abstract}
We discuss effects of fragmentation and hard gluon radiation on the signal for the pair production of the lighter scalar top eigenstate $\tilde{t}_{1}$ at $e^{+} e^{-}$colliders. The main emphasis is on scenarios with small stop-LSP mass splitting, where strong interaction effects can considerably modify kinematical properties of the final state.
\end{abstract}

The search for supersymmetry is on the "to do" list of all experiments at currently operating or planned high energy particle colliders. It is commonly believed that hadron colliders are better suited for the search for strongly interacting sparticles (gluinos and squarks) than lepton colliders are. Hadron colliders indeed offer larger cross sections for producing such sparticles. However, experiments at hadron colliders also suffer from much larger backgrounds; severe cuts are then needed to extract a signal for sparticle production 1 . As a result, the production of sparticles will become undetectable if their decays only release a small amount of visible energy.

This might well be the case for the lighter stop mass eigenstate $\tilde{t}_{1}$. It is expected to be significantly lighter than the other squarks, due to the renormalization group running of spft breaking stop masses as well as mixing between $\tilde{t}_{L}$ and $\tilde{t}_{R}$ current eigenstates 1 . In fact, $m_{\tilde{t}_{1}}$ could be quite close to the mass of the LSP, usually assumed to be the lightest neutralino $\tilde{\chi}_{1}^{0}$. In this case $\tilde{t}_{1}$ becomes quite long-lived, decaying primarily into $q \tilde{\chi}_{1}^{0}(q=u, c)$ through $\tilde{t}-\tilde{q}$ mixing $B$. Since flavor mixing in the squark sector has to be small in order to satisfy FCNC constraints, and might in fact occur only at the one-loop level, the lifetime of $\tilde{t}_{1}$ will then exceed the hadronization time scale, i.e. $\tilde{t}_{1}$ will fragment into a (spin-1/2) stop "meson" before it decays 3 .

Implementing this fragmentation in an event generator is not entirely straightforward. The reason is that fragmentation of a massive on-shell squark into a hadron with equal or greater mass violates the conservation of energy and/or 3-momentum. In ref we have developed two algorithms to solve this problem. Briefly, method I allows energy conservation to be violated in the fragmentation step, but takes care to conserve 3-momentum; overall energy conservation is then restored by rescaling all 3-momenta by a common factor, which is calculated numerically by iteration. In the second method, the stop squarks are created off-shell; the fragmentation step can then conserve both energy and 3-momentum, being formally equivalent to the (collinear) decay of a (virtual) squark with mass $m_{*}$ into a "meson" with mass $m_{\tilde{t}_{M}}<m_{*}$ and a massless (color triplet) "fragmentation jet". The distribution in $m_{*}$ is chosen such that the energy distribution of the stop "meson" resembles that predicted by the assumed fragmentation function. 
A second ambiguity is related to the choice of the fragmentation variable $x$. For fixed functional form of the fragmentation function $D_{\tilde{t}}(x)$, defining $x$ as the ratio of "meson" and squark energies (in the lab frame) will give a much softer stop "meson" energy spectrum than defining $x$ as the ratio of "meson" and squark 3 -momenta. Fortunately we found 4 that much, although not all, of the differences between the stop "meson" energy spectra predicted by our two different algorithms for stop fragmentation, or using different definitions of the fragmentation variable $x$, can be absorbed in a re-definition of the single parameter $\epsilon$ of the Peterson et al. fragmentation function 1 .

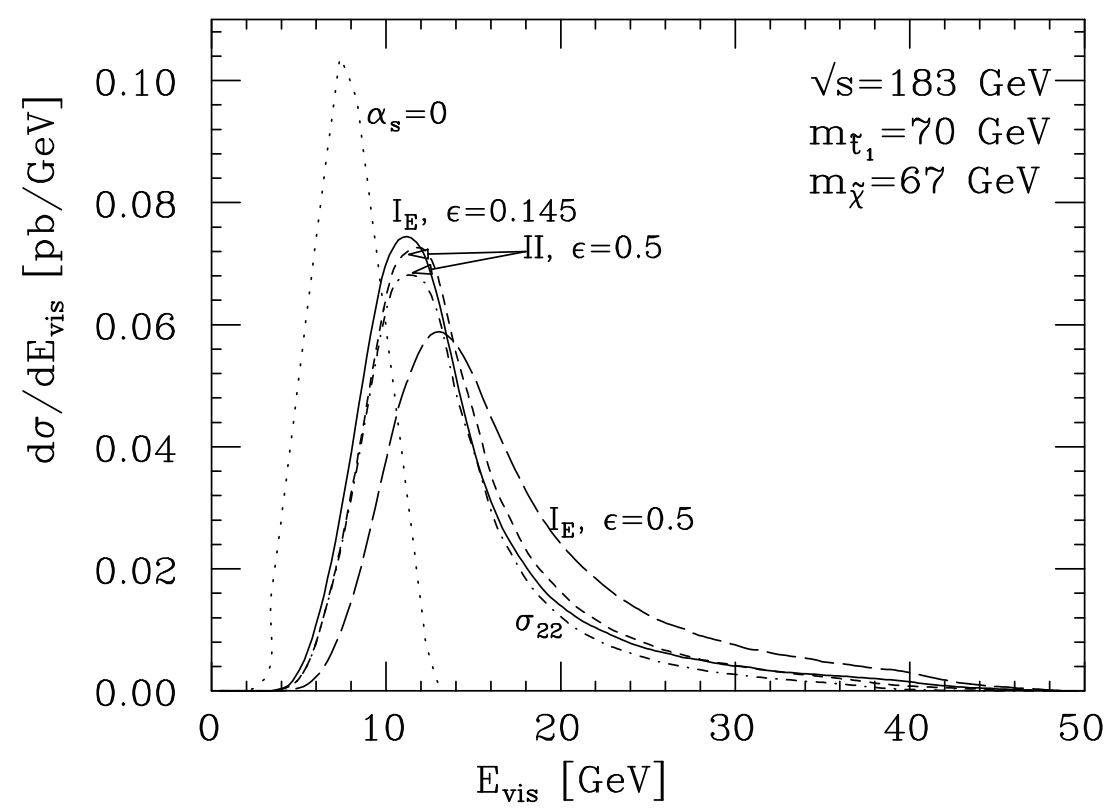

Figure 1: The total visible energy in $e^{+} e^{-} \rightarrow \tilde{t}_{1} \tilde{t}_{1}^{*}(g)$ events at LEP-183. The dotted curve has been obtained by switching off both perturbative and non-perturbative strong interaction effects, while the dot-dashed curve includes non-perturbative $\tilde{t}_{1}$ fragmentation but no hard gluon radiation. The other curves include both perturbative and non-perturbative effects. The labels refer to the fragmentation scheme used, and give the value of the fragmentation parameter $\epsilon$ in units of $\mathrm{GeV}^{2}$

Additional contributions to the visible energy can come from perturbative QCD processes. In particular, the $\tilde{t}_{1}$ pair can be produced in association with a hard gluon 1 . As shown in Fig. 1, this perturbative contribution to the visible energy is actually smaller than the non-perturbative one, at least for beam energies not far above the $\tilde{t}_{1}$ pair threshold. It nevertheless plays an important role in searches for $\tilde{t}_{1}$ production in scenarios with very small $\tilde{t}_{1}-\tilde{\chi}_{1}^{0}$ mass difference $\Delta m$. In the 
absence of hard gluon radiation the final state then consists essentially of the two fragmentation jets, which are back-to-back in the transverse plane. Such final states suffer very large backgrounds from two-photon processes. Experimental searches for $\tilde{t}_{1}$ production at LEP therefore require the final state to have an acoplanarity angle significantly smaller than $180^{\circ}$. This becomes possible in the presence of hard gluon radiation even if $\Delta m \rightarrow 0$. As shown in Fig. 2, $\tilde{t}_{1} \tilde{t}_{1}^{*} g$ events should therefore allow to extend LEP searches to the case $\Delta m \rightarrow 0$, although the reach in $m_{\tilde{t}_{1}}$ will be smaller than for larger values of $\Delta m$.

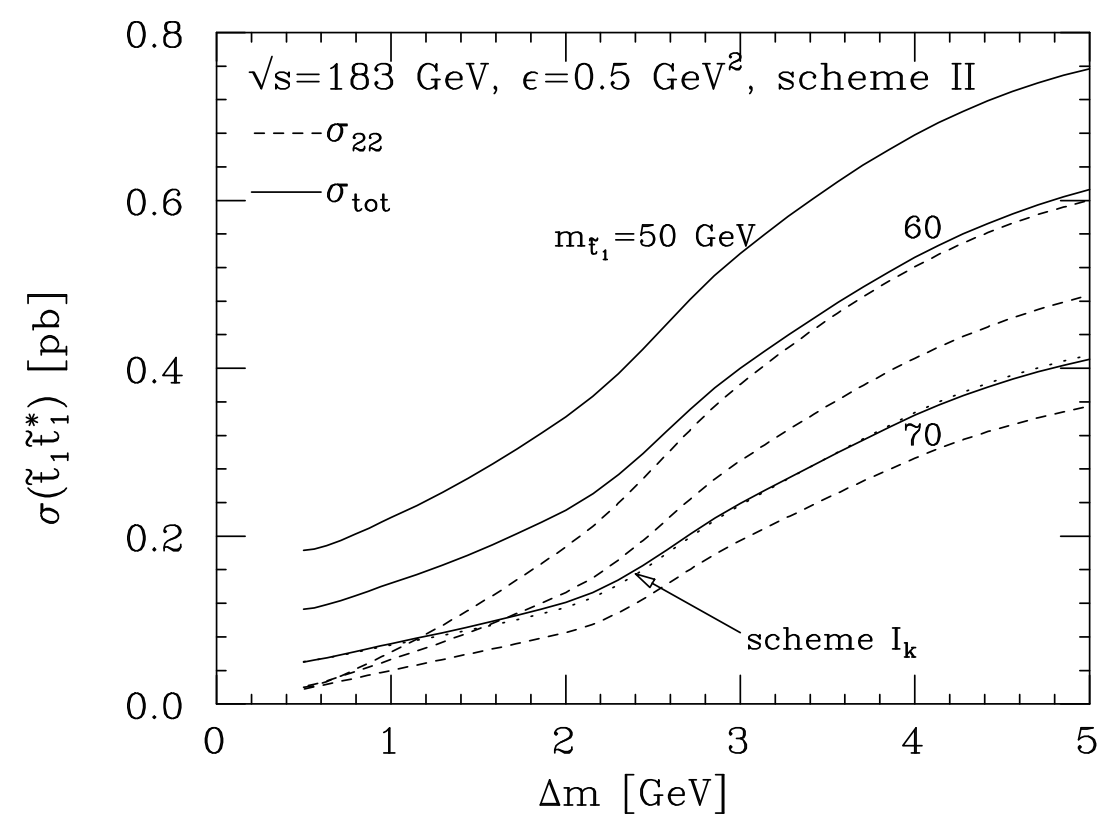

Figure 2: The total inclusive $\tilde{t}_{1} \tilde{t}_{1}^{*}$ cross section at LEP-183 after cuts similar to those used by the OPAL collaboration have been applied. The solid curves show the full result, while the dashed curves have been computed by switching off hard gluon radiation. All curves are for fragmentation scheme II, except the dotted one, which is for scheme $\mathrm{I}_{k}$.

Analogous results should hold for $\tilde{t}_{1}$ searches at future linear colliders. However, there the size of the two-photon background, and hence the potential for $\tilde{t}_{1}$ searches in scenarios with small $\Delta m$, depends quite sensitively on details of the design of both the accelerator and the detector. The former determines the flux of beamstrahlung photons, which can easily dominate the total effective photon flux in the relevant energy range of a few $\mathrm{GeV}$. The latter will e.g. determine the minimal angle at which outgoing $e^{ \pm}$can be vetoed, and hence the maximal missing transverse momentum in two-photon events. We therefore do not attempt to repeat an analysis like that shown in Fig. 2 for higher energies. 
Finally, we remark that QCD corrections to $\tilde{t}_{1}$ decay were not included in our results. An accurate treatment of these decays is not very important if $\Delta m$ is small, since kinematical constraints then imply that the contribution to the visible energy from these decays is in any case small. This is fortunate, since for $\Delta m \leq 2 \mathrm{GeV}$ a perturbative treatment of these decays becomes altogether unreliable. On the other hand, QCD effects are expected to be important also for the interpretation of precision measurements of squark properties, in particular for the determination of their masses, even if $\Delta m$ is large; the possibility to perform such precision studies is another major selling point of $e^{+} e^{-}$colliders. In this case a careful treatment of squark decays, including QCD forrections, becomes important. An investigation of these effects is now in progress $⿴$.

\section{Acknowledgements}

This work was supported in part by Conselho Nacional de Desenvolvimento Científico e Tecnológico $(\mathrm{CNPq})$, by Fundação de Amparo à Pesquisa do Estado

de São Paulo (FAPESP), and by Programa de Apoio a Núcleos de Excelência (PRONEX).

\section{References}

1. See e.g. H. Baer et al., hep-ph/9503479, in Electroweak Symmetry Breaking and New Physics at the TeV Scale, eds. T. L. Barklow, S. Dawson, H. E. Haber and J. L. Siegrist, World Scientific (1996).

2. J. Ellis and S. Rudaz, Phys. Lett. B128, 248 (1983).

3. K. Hikasa and M. Kobayashi, Phys. Rev. D36, 724 (1987); C. Boehm, A. Djouadi and Y. Mambrini, hep-ph/9907428.

4. M. Drees and O. Éboli, hep-ph/9902391, to appear in Eur. Phys. J. C.

5. C. Peterson, D. Schlatter, I. Schmitt, and P.M. Zerwas, Phys. Rev. D27, 105 (1983).

6. W. Beenakker, R. Höpker, and P.M. Zerwas, Phys. Lett. B349, 463 (1995), hep-ph/9501292; A. Arhrib, M. Capdequi-Peyranere and A. Djouadi, Phys. Rev. D52, 1404 (1995), hep-ph/9412382; K. Hikasa and J. Hisano, Phys. Rev. D54, 1908 (1996), hep-ph/9603203.

7. ALEPH collab., R. Barate et al., Phys. Lett. B413, 431 (1997), and Phys. Lett. B434, 189 (1998), hep-ex/9810028; DELPHI collab., P. Abreu et al., Eur. Phys. J. C6, 385 (1999); OPAL collab., K. Ackerstaff et al., Eur. Phys. J. C6, 225 (1999), hep-ex/9808026.

8. M. Drees, O. Éboli, R. Godbole, and S. Kraml, in preparation. 